Type of the Paper (Article)

\title{
Hematological and biochemical reference ranges for population with sickle cell disease at steady-state in Tanzania
}

\author{
Anna Daniel Fome ${ }^{1,2,}$, , Raphael Zozimus Sangeda ${ }^{1,3}$, Josephine Mgayaa ${ }^{1}$, Deogratius Soka ${ }^{1}$, Furahini Tluway ${ }^{1}$, Upendo \\ Masamu ${ }^{1}$ Emmanuel Balandya ${ }^{1}$, Siana Nkya ${ }^{1}$, Julie Makani1 ${ }^{1,4}$ and Bruno P. Mmbando ${ }^{1,5}$
}

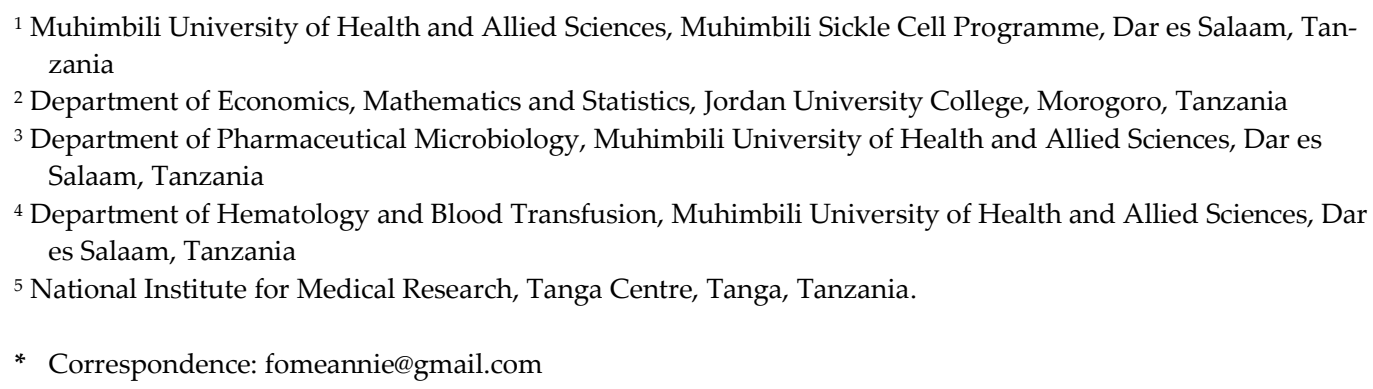

\begin{abstract}
Hematological and biochemical reference values in sickle cell disease (SCD) are crucial for patient management and evaluation of interventions. This study was conducted at Muhimbili National Hospital (MNH) in Dar es Salaam, to establish laboratory reference ranges in SCD at steady-state. Patients were grouped into five age groups with respects to their sex. Aggregate functions were used to handle repeated measures within the individual level in each age group. A nonparametric approach was used to smooth the curves and a parametric approach was used to determine SCD normal ranges. Comparison between males and females and against the general population was documented. Data from 4,422 patients collected from 2004-2015 were analyzed. The majority of the patients (35.41\%) were children aged between 5-11 years. There were no significant differences $(\mathrm{p} \geq 0.05)$ in mean corpuscular hemoglobin concentration (MCHC), lymphocytes, basophils and bilirubin direct observed between males and females. Significant differences $(\mathrm{p}<0.05)$ were observed in all selected parameters across age groups except neutrophils and MCHC in adults, as well as platelets and alkaline phosphatase in infants when SCD estimates were compared to the general population. Laboratory reference ranges in SCD at steady-state were different from those of the general population and varied with sex and age. The established reference ranges for SCD at steady-state will be a helpful in the management and monitoring of the progress of SCD.
\end{abstract}

Keywords: Hematological parameters; biochemical parameters; reference ranges; sickle cell disease; Tanzania; steady-state.

\section{Introduction}

Sickle cell disease (SCD) is the most common single-gene hemoglobin disorder with a global estimate of 330,000 birth affected annually [1] . The condition results in a tremendous burden on African countries with limited resources and expertise. Approximately 280,000 newborns are affected 
each year [2]. It is estimated that $80 \%$ of children die of the SCD due to suboptimal health care services [2-4]. SCD can be managed through public health education, early diagnosis (including newborn screening), and therapeutic services to prevent infections, anemia and other sickle cell crises [4]. Bone marrow transplantation of hematopoietic stem cells has been shown to cure SCD despite the complication of the procedures, high costs and limited facilities that are available mainly in high-income countries $[5$, 6].

SCD primarily affects red blood cells but also causes changes in other hematological parameters like white blood cells and platelets $[7,8]$. Individuals with SCD go through periods characterized by steady-state and crises which associated with sickle cell complications such as anemia and pain [9]. These generally affect the levels of hematological parameters. Hematological levels below the reference values are associated with mild to severe health conditions. While the low neutrophil count has been associated with a good prognosis in SCD patients, many complications have been associated with leukocytes [7].

Furthermore, research has shown that patients with high leukocyte count, low steady-state hemoglobin and lower fetal hemoglobin level $(\mathrm{HbF})$ have increased risks for sickle cell crises, including stroke [10]. Although there is a scarcity of hematological and biochemical parameters in most African countries [11-13], the significance of these laboratory reference values for SCD has received considerable attention in recent years [7, $8,14-16]$. Reference values are widely used in medical decision-making. Only a few laboratories have their reference interval established from their population. Most other laboratories rely on reference values created many years ago and those generated from a different population whose characteristics are likely to be very different [17].

While most hematological and biochemical majority parameters in individuals with SCD are different from that of general the population, understanding the reference values for individuals with SCD is essential to monitor patients' progress, especially compared to those in the steady-state. Steady-state was defined as the point in time where SCD patient was in good health (absence of clinical events) and had not received treatment with medications such as antibiotics that could have affected blood count during three previous weeks. Besides, no history of blood transfusion during the last four months and without the history of acute pain that required treatment in the emergency department within one month before the observation $[9,18]$.

\section{Materials and Methods}

This study utilized data collected from a prospective cohort study of SCD conducted at Muhimbili National Hospital, Tanzania [19] to establish the hematological and biochemical reference values of SCD at steady-state. 


\subsection{Patients}

Hematological and biochemical data were collected longitudinally from 2004 to 2015. Data on 4,422 SCD patients with confirmed HbSS and 15,461 visits were available for analysis; all the other hemoglobin genotypes were excluded. Patients were categorized into adults and pediatrics (infants, children, and adolescents) groups. The age groups were divided into $<1$ year (infants), 1-4 years, and 5-11 years (children), 12-17 years (adolescents), and those aged from 18 years and above (adults). Only records of patients at steady-state visits were included in the current study.

\subsection{Hematological and biochemical parameters}

Twenty-two laboratory parameters were used in this study. Among those sixteen are hematological parameters; hemoglobin $(\mathrm{Hb})$ count, fetal hemoglobin $(\mathrm{HbF})$, mean corpuscular hemoglobin $(\mathrm{MCH})$, mean corpuscular hemoglobin concentration (MCHC). Other parameters include the red blood cell (RBC) count, mean corpuscular volume (MCV), red cell distribution width (RDW), reticulocyte count, platelet count, and mean platelet volume (MPV). Besides, white blood cells (WBC), neutrophils, lymphocytes, eosinophils, monocytes, and basophils are measured. The other six are biochemical parameters, which include bilirubin total, bilirubin direct, aspartate aminotransferase (AST), alkaline phosphatase (ALP), lactate dehydrogenase $(\mathrm{LDH})$ and creatinine.

\subsection{Statistical analysis}

We assessed data for outliers using box-and-whisker plots. Values below the lowest and above the highest observations were considered as outliers and wereexcluded from the analysis. Repeated measurements within the individual level were collapsed by calculating the mean value for everyone within the age range using an aggregate function in R. Variables were tested for a normal distribution using diagnostic plots (i.e., Q-Q plots, boxplots and histograms), Shapiro-Wilk test and AndersonDarling test. Some variables were transformed using log and square root for those that didn't pass normality tests. Upper and lower normal values were determined using the mean plus or minus 1.96 standard deviations [20]. The sample population was grouped by sex for each age category. Mean differences between males and females and against the general population were evaluated using the t-test and Mann Whitney U test, respectively. Loess regression was used to smooth curves for all parameters which were identified to be significantly different. All ages above 35 were rounded to 35 years to restrict fewer observations in higher ages. A p-value of less than 0.05 was considered significant.

\section{Results}

\subsection{Demographic characteristics}

A total of 4,422 SCD patients (2,235, 50.5\% females) were included in this study. Females were significantly older than males $(2,187,49.5 \%)$. The mean difference 
across the age groups was not significant except in the age group 1-4 years. The majority $(1566,35.41 \%)$ of patients were children aged between $5-11$ years, while a minority $(135,3.05 \%$ of patients) were infants, as presented in Table 1.

Table 1. Demographic parameters of SCD Patients. Means values are obtained after finding the mean ages within the individual level for each group.

\begin{tabular}{|l|l|l|l|l|}
\hline Parameters & $\begin{array}{l}\text { Females (Mean age } \pm \\
\text { SD) }\end{array}$ & $\begin{array}{l}\text { Males (Mean age } \pm \\
\text { SD) }\end{array}$ & $\begin{array}{l}\text { P-values* } \\
\text { Overall } \\
\text { Mean age } \pm \\
\text { SD }\end{array}$ \\
\hline Infants (<1) & $0.69 \pm 0.26$ & $0.72 \pm 0.21$ & 0.426 & $0.71 \pm 0.23$ \\
\hline Frequency & $59(1.33)$ & $76(1.72)$ & & $135(3.05)$ \\
\hline Children (1-4) & $3.39 \pm 1.03$ & $3.25 \pm 1.00$ & 0.023 & $3.3 \pm 1.02$ \\
\hline Frequency (\%) & $478(10.80)$ & $520(11.75)$ & & $998(22.6)$ \\
\hline Children (5-11) & $8.44 \pm 1.90$ & $8.29 \pm 1.89$ & 0.144 & $8.36 \pm 1.90$ \\
\hline Frequency (\%) & $766(17.32)$ & $800(18.09)$ & & $1566(35.41)$ \\
\hline $\begin{array}{l}\text { Adolescent (12- } \\
17)\end{array}$ & $14.74 \pm 1.59$ & $14.59 \pm 1.55$ & 0.125 & $14.66 \pm 1.57$ \\
\hline Frequency (\%) & $476(10.76)$ & & & $956(21.61)$ \\
\hline Adults $(\geq 18)$ & $23.61 \pm 5.28$ & $480(10.85)$ & & $23.36 \pm 5.22$ \\
\hline Frequency & $456(10.31)$ & $23.0 \pm 5.11$ & & $767(17.34)$ \\
\hline Total & $11.59 \pm 7.75$ & $311(7.03)$ & & $10.95 \pm 7.42$ \\
\hline Frequency (\%) & $2235(50.54)$ & $10.30 \pm 7.01$ & $2187(49.46)$ & $4422(100.0)$ \\
\hline
\end{tabular}

*Welch Two Sample t-test for the mean difference.

\subsection{Comparison of laboratory parameters in $S C D$}

Table 2 and Table 3 displays the means and reference intervals for the study population stratified by age groups and sex and by combined sex across different age groups. Where the mean values by sex are not different (i.e., $\mathrm{p} \geq 0.05$ ), the ranges combining females and males are considered. Otherwise, sex-specific ranges are considered. Figures 1 and 2 represents smoothed means curves of parameters statistically different between males and females. From the figures, the bandwidth represents a $95 \%$ confidence interval. Therefore, there is a 0.95 probability that the true regression line for the population will lie within the confidence interval.

Table 2 Steady-state hematological reference values calculated as mean (mean $\pm 1.965 D$ ) in SCD categorized by age groups and sex.

\begin{tabular}{|c|c|c|c|c|c|c|c|}
\hline \multirow{2}{*}{$\begin{array}{l}\text { Age } \\
\text { group } \\
\text { (years) }\end{array}$} & \multicolumn{2}{|r|}{ Females } & \multicolumn{2}{|r|}{ Males } & \multicolumn{2}{|r|}{ Combined } & \multirow{2}{*}{ P-values* } \\
\hline & $\mathbf{n}$ & $\begin{array}{c}\text { Mean (Mean } \\
\pm 1.96 \mathrm{SD})\end{array}$ & $\mathbf{n}$ & Mean (Mean $\pm 1.96 S D)$ & $\mathbf{n}$ & Mean (Mean $\pm 1.96 S D)$ & \\
\hline \multicolumn{8}{|c|}{ Hemoglobin (g/dL) } \\
\hline$<1$ & 59 & $7.3(4.8-9.8)$ & 76 & $7.2(4.5-9.8)$ & 135 & $7.2(4.6-9.8)$ & 0.4574 \\
\hline
\end{tabular}




\begin{tabular}{|c|c|c|c|c|c|c|c|}
\hline \multirow{2}{*}{$\begin{array}{l}\text { Age } \\
\text { group } \\
\text { (years) }\end{array}$} & \multicolumn{2}{|r|}{ Females } & \multicolumn{2}{|r|}{ Males } & \multicolumn{2}{|r|}{ Combined } & \multirow{2}{*}{ P-values* } \\
\hline & $\mathbf{n}$ & $\begin{array}{c}\text { Mean (Mean } \\
\pm 1.96 \mathrm{SD})\end{array}$ & $\mathbf{n}$ & Mean (Mean $\pm 1.96 S D)$ & $\mathbf{n}$ & Mean $($ Mean $\pm 1.96 S D)$ & \\
\hline $1-4$ & 477 & 7.4(5.0-9.7) & 518 & 7.2(4.7-9.6) & 995 & 7.3(4.9-9.7) & 0.0128 \\
\hline 5-11 & 766 & 7.4(5.1-9.6) & 799 & 7.2(5.1-9.3) & 1565 & $7.3(5.1-9.5)$ & 0.0018 \\
\hline $12-17$ & 476 & 7.6(5.4-9.7) & 480 & 7.4(5.1-9.7) & 956 & 7.5(5.3-9.7) & 0.0171 \\
\hline$\geq 18$ & 456 & 7.6(5.1-10.0) & 310 & 8.2(5.0-11.3) & 766 & 7.8(5.0-10.6) & $<0.0001$ \\
\hline \multicolumn{8}{|c|}{$\mathrm{HbF}(\%)$} \\
\hline$<1$ & 29 & $16.5(4.0-67.7)$ & 42 & $16.0(3.1-81.2)$ & 71 & $16.2(3.5-75.2)$ & 0.7882 \\
\hline $1-4$ & 278 & $9.8(2.7-35.4)$ & 272 & $9.3(2.9-29.5)$ & 550 & $9.6(2.8-32.4)$ & 0.1696 \\
\hline 5-11 & 379 & 6.6(1.9-23.1) & 377 & $5.5(1.5-19.9)$ & 756 & $6.0(1.6-21.8)$ & 0.0006 \\
\hline $12-17$ & 150 & 5.8(1.3-24.4) & 135 & $4.9(1.3-18.0)$ & 285 & $5.3(1.3-21.4)$ & 0.0341 \\
\hline$\geq 18$ & 162 & $5.9(1.5-22.5)$ & 103 & $3.9(0.9-16.6)$ & 265 & $5.0(1.2-21.1)$ & 0.0005 \\
\hline \multicolumn{8}{|c|}{ MCH (pg) } \\
\hline$<1$ & 58 & 23.4(17.4-29.4) & 75 & $22.4(14.9-30.0)$ & 133 & $22.9(15.9-29.8)$ & 0.1109 \\
\hline $1-4$ & 476 & $25.0(18.7-31.3)$ & 514 & 24.5(18.2-30.9) & 990 & 24.8(18.4-31.1) & 0.0357 \\
\hline $5-11$ & 762 & 25.8(19.0-32.6) & 798 & 25.4(18.8-32.1) & 1560 & 25.6(18.9-32.4) & 0.0392 \\
\hline $12-17$ & 476 & $26.8(20.2-33.4)$ & 478 & 25.9(19.2-32.7) & 954 & $26.4(19.6-33.1)$ & $<0.0001$ \\
\hline$\geq 18$ & 453 & 27.6(20.3-34.9) & 311 & 26.5(19.6-33.5) & 764 & $27.2(20.0-34.4)$ & $<0.0001$ \\
\hline \multicolumn{8}{|c|}{ MCHC (g/dL) } \\
\hline$<1$ & 58 & $31.0(28.6-33.5)$ & 76 & $30.9(27.4-34.4)$ & 134 & $31.0(27.9-34.0)$ & 0.5994 \\
\hline $1-4$ & 477 & $31.4(28.1-34.7)$ & 518 & $31.4(28.0-34.9)$ & 995 & $31.4(28.0-34.8)$ & 0.9934 \\
\hline $5-11$ & 765 & 32.1(28.9-35.4) & 799 & $32.1(28.6-35.6)$ & 1564 & $32.1(28.7-35.5)$ & 0.8559 \\
\hline $12-17$ & 476 & $32.8(29.5-36.1)$ & 478 & $32.9(29.6-36.1)$ & 954 & $32.8(29.6-36.1)$ & 0.5454 \\
\hline$\geq 18$ & 456 & $32.9(29.8-35.9)$ & 311 & $32.9(29.7-36.1)$ & 767 & $32.9(29.8-36.0)$ & 0.9422 \\
\hline \multicolumn{8}{|c|}{ RBC Count $\left(\times 10^{12} / 1\right)$} \\
\hline$<1$ & 59 & $3.2(1.7-4.6)$ & 76 & 3.3.(1.4-5.2) & 135 & $3.2(1.5-4.9)$ & 0.4144 \\
\hline $1-4$ & 478 & 3.0(1.6-4.4) & 518 & 3.0(1.5-4.4) & 996 & 3.0(1.6-4.4) & 0.3762 \\
\hline $5-11$ & 766 & 2.9(1.6-4.2) & 800 & 2.8(1.5-4.2) & 1566 & $2.9(1.6-4.2)$ & 0.2956 \\
\hline $12-17$ & 476 & $2.8(1.7-4.0)$ & 478 & $2.9(1.5-4.3)$ & 954 & $2.9(1.6-4.2)$ & 0.2519 \\
\hline$\geq 18$ & 456 & 2.8(1.5-4.1) & 310 & 3.1(1.4-4.9) & 766 & $2.9(1.4-4.5)$ & $<0.0001$ \\
\hline \multicolumn{8}{|c|}{ MCV (fL) } \\
\hline$<1$ & 58 & $75.4(57.8-92.9)$ & 76 & 72.7(50.9-94.4) & 134 & $73.8(53.7-94.0)$ & 0.2167 \\
\hline $1-4$ & 477 & 79.4(61.2-97.7) & 517 & 78.1(59.9-96.2) & 994 & $78.7(60.5-97.0)$ & 0.0106 \\
\hline 5-11 & 765 & $80.3(60.7-99.9)$ & 799 & 79.2(60.7-97.7) & 1564 & $79.7(60.7-98.8)$ & 0.0140 \\
\hline $12-17$ & 476 & $81.8(64.0-99.5$ & 478 & $78.9(60.2-97.5)$ & 954 & $80.3(61.9-98.8)$ & $<0.0001$ \\
\hline$\geq 18$ & 455 & 83.9(64.1-103.7) & 311 & $80.7(61.5-99.9)$ & 766 & $82.6(62.8-102.4)$ & $<0.0001$ \\
\hline \multicolumn{8}{|c|}{ RDW (\%) } \\
\hline$<1$ & 59 & 23.7(16.9-30.5) & 75 & $24.3(17.4-31.3)$ & 134 & 24.1(17.2-30.9) & 0.1860 \\
\hline $1-4$ & 476 & $22.6(16.2-28.9)$ & 518 & 23.3(16.9-29.7) & 994 & $22.9(16.5-29.4)$ & $<0.0001$ \\
\hline
\end{tabular}




\begin{tabular}{|c|c|c|c|c|c|c|c|}
\hline \multirow{2}{*}{\begin{tabular}{|l} 
Age \\
group \\
(years)
\end{tabular}} & \multicolumn{2}{|r|}{ Females } & \multicolumn{2}{|r|}{ Males } & \multicolumn{2}{|r|}{ Combined } & \multirow{2}{*}{ P-values* } \\
\hline & $\mathbf{n}$ & $\begin{array}{c}\text { Mean (Mean } \\
\pm 1.96 \mathrm{SD})\end{array}$ & $\mathbf{n}$ & Mean (Mean $\pm 1.96 S D)$ & $\mathbf{n}$ & Mean $($ Mean $\pm 1.96 \mathrm{SD})$ & \\
\hline 5-11 & 764 & 21.6(15.4-27.8) & 800 & $22.3(16.3-28.4)$ & 1564 & $22.0(15.8-28.1)$ & $<0.0001$ \\
\hline $12-17$ & 476 & $21.2(15.0-27.5)$ & 478 & 21.9(16.1-27.6) & 956 & 21.6(15.5-27.6) & $<0.0001$ \\
\hline$\geq 18$ & 456 & $20.3(14.5-26.1)$ & 310 & 21.6(15.6-27.6) & 766 & $20.8(14.8-26.8)$ & $<0.0001$ \\
\hline \multicolumn{8}{|c|}{ Reticulocyte Count (\%) } \\
\hline$<1$ & 20 & $12.1(3.0-27.2)$ & 17 & $13.0(3.2-29.4)$ & 37 & $12.5(3.2-28.0)$ & 0.7032 \\
\hline $1-4$ & 201 & $13.4(3.6-29.3)$ & 221 & $12.5(2.0-31.9)$ & 422 & $12.9(2.7-30.7)$ & 0.4093 \\
\hline $5-11$ & 336 & $12.4(3.8-26.0)$ & 365 & 12.1(3.6-25.6) & 701 & $12.3(3.7-25.8)$ & 0.9524 \\
\hline $12-17$ & 235 & $12.0(3.8-24.7)$ & 198 & $11.1(3.4-23.3)$ & 433 & $11.6(3.6-24.1)$ & 0.0894 \\
\hline$\geq 18$ & 202 & $11.9(3.9-24.2)$ & 122 & $11.0(3.9-21.8)$ & 324 & 11.6(3.9-23.4) & 0.0411 \\
\hline \multicolumn{8}{|c|}{ Platelet Count $\left(\times 10^{9} / 1\right)$} \\
\hline$<1$ & 59 & 397.9(73.3-722.4) & 76 & $410.8(56.8-764.7)$ & 135 & $405.1(64.8-745.5)$ & 0.7935 \\
\hline $1-4$ & 476 & 375.1(55.8-694.4) & 517 & 399.2(67.0-731.4) & 993 & 387.7(60.9-714.4) & 0.0183 \\
\hline 5-11 & 766 & 422.3(110.9-733.6) & 798 & $440.2(103.8-776.6)$ & 1564 & $431.4(106.7-756.2)$ & 0.1210 \\
\hline $12-17$ & 476 & $447.0(130.6-763.4)$ & 477 & 453.3(142.5-764.1) & 953 & $450.2(136.7-763.7)$ & 0.9320 \\
\hline$\geq 18$ & 456 & $442.9(149.5-736.3)$ & 309 & $447.0(149.2-744.9)$ & 765 & $444.6(149.6-739.6)$ & 0.7580 \\
\hline \multicolumn{8}{|c|}{ MPV (fL) } \\
\hline$<1$ & 53 & 8.5(6.8-9.9) & 68 & 8.3(6.4-10.5) & 121 & $8.4(6.5-10.2)$ & 0.9604 \\
\hline $1-4$ & 458 & 8.7(6.9-10.3) & 498 & $8.6(6.8-10.1)$ & 956 & $8.6(6.9-10.2)$ & 0.2977 \\
\hline 5-11 & 741 & 8.6(7.0-10.2) & 781 & 8.4(6.8-10.1) & 1522 & $8.6(6.9-10.2)$ & 0.0002 \\
\hline $12-17$ & 467 & 8.5(6.9-10.4) & 470 & $8.5(7.0-10.3)$ & 937 & $8.5(7.0-10.4)$ & 0.7275 \\
\hline$\geq 18$ & 443 & 8.5(7.1-10.5) & 304 & 8.6(7.1-10.6) & 747 & 8.6(7.1-10.5) & 0.3750 \\
\hline \multicolumn{8}{|c|}{ WBC $\left(\times 10^{9} / 1\right)$} \\
\hline$<1$ & 57 & $16.4(3.8-29.1)$ & 72 & 16.6(1.3-31.3) & 129 & $16.4(2.4-30.3)$ & 0.4381 \\
\hline $1-4$ & 473 & $17.1(6.5-27.6)$ & 518 & 18.2(7.3-29.2) & 991 & $17.7(6.9-28.5)$ & 0.0006 \\
\hline $5-11$ & 798 & $14.7(5.6-23.8)$ & 766 & $15.7(7.1-24.4)$ & 1564 & $15.2(6.3-24.1)$ & $<0.0001$ \\
\hline $12-17$ & 476 & $12.5(5.8-19.2)$ & 477 & $13.3(6.0-20.7)$ & 953 & $12.9(5.9-20.0)$ & 0.0003 \\
\hline$\geq 18$ & 455 & 11.6(4.7-18.4) & 311 & $11.9(4.3-19.6)$ & 766 & $11.7(4.5-18.9)$ & 0.4009 \\
\hline \multicolumn{8}{|c|}{ Neutrophils (\%) } \\
\hline$<1$ & 56 & $27.2(7.0-47.3)$ & 69 & 29.3(8.2-50.4) & 125 & $28.3(7.6-49.1)$ & 0.1985 \\
\hline $1-4$ & 447 & $36.5(18.1-55.0)$ & 482 & $36.7(18.1-55.2)$ & 929 & $36.6(18.1-55.1)$ & 0.9277 \\
\hline 5-11 & 724 & $41.6(25.2-58.0)$ & 783 & 42.2(25.4-58.9) & 1507 & $41.9(25.3-58.5)$ & 0.2629 \\
\hline $12-17$ & 465 & $45.5(29.1-62.0)$ & 460 & $44.0(27.6-60.5)$ & 925 & $44.8(28.3-61.3)$ & 0.0091 \\
\hline$\geq 18$ & 449 & $48.5(28.9-68.0)$ & 305 & $46.9(25.5-68.1)$ & 754 & $47.8(27.6-68.1)$ & 0.0132 \\
\hline \multicolumn{8}{|c|}{ Lymphocytes (\%) } \\
\hline$<1$ & 59 & $56.6(29.7-83.5)$ & 75 & 53.3(29.3-77.4) & 134 & $54.8(29.3-80.2)$ & 0.0740 \\
\hline $1-4$ & 472 & $49.4(29.4-69.5)$ & 516 & $48.6(28.2-69.1)$ & 988 & $49.0(28.8-69.3)$ & 0.2216 \\
\hline $5-11$ & 763 & $43.9(26.7-61.1)$ & 796 & $43.0(26.2-59.8)$ & 1559 & $43.5(26.4-60.5)$ & 0.0612 \\
\hline
\end{tabular}




\begin{tabular}{|c|c|c|c|c|c|c|c|}
\hline \multirow{2}{*}{$\begin{array}{l}\text { Age } \\
\text { group } \\
\text { (years) }\end{array}$} & \multicolumn{2}{|r|}{ Females } & \multicolumn{2}{|r|}{ Males } & \multicolumn{2}{|r|}{ Combined } & \multirow{2}{*}{ P-values* } \\
\hline & $\mathbf{n}$ & $\begin{array}{c}\text { Mean (Mean } \\
\pm 1.96 \mathrm{SD})\end{array}$ & $\mathbf{n}$ & Mean (Mean $\pm 1.96 S D$ ) & $\mathbf{n}$ & Mean $($ Mean $\pm 1.96 \mathrm{SD})$ & \\
\hline $12-17$ & 474 & $40.1(24.1-56.1)$ & 476 & $39.6(23.6-55.7)$ & 950 & 39.9(23.9-55.9) & 0.3484 \\
\hline$\geq 18$ & 454 & $37.0(20.5-53.5)$ & 310 & $36.5(17.5-55.5)$ & 764 & $36.8(19.2-54.3)$ & 0.4455 \\
\hline \multicolumn{8}{|c|}{ Monocytes (\%) } \\
\hline$<1$ & 57 & 8.8(3.3-23.4) & 74 & $9.7(3.7-25.7)$ & 131 & $9.3(3.5-24.7)$ & 0.1492 \\
\hline $1-4$ & 466 & 8.3(3.7-18.6) & 507 & 8.8(3.7-20.5) & 973 & 8.6(3.7-19.6) & 0.0252 \\
\hline 5-11 & 756 & 8.7(4.2-18.1) & 793 & 8.9(4.1-19.0) & 1549 & 8.8(4.1-18.5) & 0.3807 \\
\hline $12-17$ & 473 & $9.4(4.6-19.2)$ & 472 & $9.9(4.8-20.4)$ & 945 & $9.7(4.7-19.8)$ & 0.0121 \\
\hline$\geq 18$ & 449 & $9.5(4.7-19.0)$ & 306 & $10.5(5.0-22.2)$ & 755 & $9.9(4.8-20.4)$ & $<0.0001$ \\
\hline \multicolumn{8}{|c|}{ Eosinophils (\%) } \\
\hline$<1$ & 59 & $3.7(1.2-11.5)$ & 72 & 3.4(1.1-10.3) & 131 & 3.5(1.1-10.8) & 0.4710 \\
\hline $1-4$ & 462 & $3.5(1.2-9.8)$ & 501 & 3.5(1.2-9.7) & 963 & 3.5(1.2-9.8) & 0.8414 \\
\hline 5-11 & 743 & 3.8(1.3-11.4) & 790 & 4.0(1.4-11.5) & 1533 & $3.9(1.3-11.5)$ & 0.1706 \\
\hline $12-17$ & 470 & 3.6(1.3-9.9) & 463 & $4.5(1.5-12.8)$ & 933 & 4.0(1.4-11.5) & $<0.0001$ \\
\hline$\geq 18$ & 454 & 3.2(1.1-8.9) & 307 & 3.8(1.2-12.2) & 761 & 3.5(1.1-10.3) & $<0.0001$ \\
\hline \multicolumn{8}{|c|}{ Basophils (\%) } \\
\hline$<1$ & 58 & $2.5(1.3-5.0)$ & 72 & 2.3(0.9-5.5) & 130 & $2.4(1.1-5.3)$ & 0.1711 \\
\hline $1-4$ & 471 & 2.1(1.2-3.8) & 510 & 2.1(1.2-3.8) & 981 & 2.1(1.2-3.8) & 0.6519 \\
\hline 5-11 & 754 & 2.0(1.1-3.4) & 795 & 2.0(1.2-3.4) & 1549 & 2.0(1.1-3.4) & 0.9251 \\
\hline $12-17$ & 473 & $1.9(1.1-3.4)$ & 474 & 1.9(1.1-3.5) & 947 & 1.9(1.1-3.5) & 0.9398 \\
\hline$\geq 18$ & 456 & $1.9(1.2-3.0)$ & 310 & 1.9(1.1-3.4) & 766 & $1.9(1.1-3.1)$ & 0.2761 \\
\hline
\end{tabular}

*Welch Two Sample t-test. SD; Standard deviation

\subsection{Haematological parameter}

The mean values of hematological indices were shown in Table 2. The levels of hemoglobin and $\mathrm{MCH}$ were significantly different in all age groups except in infants where the results were analyzed by gender. The mean $\mathrm{HbF}$ was higher in females, but the difference was statistically significant in children aged 5-11 years, adolescence and adults (p-values of 0.0006, 0.0341, and 0.0005, respectively). While MCHC slightly increased with age, the mean levels were comparable between females and males. RBCs and reticulocyte count were significantly higher levels in males aged $\geq 18$ years, with $p$-values of $<0.0001$ and 0.0411, respectively. The mean MCV and RDW in the infants group were alike when the result was examined by gender. Platelet count mean levels were lower in females, but the difference was 
merely evidently in children aged $1-4$ years $(p=0.0183)$. MPV was significantly higher in female children aged 5-11 years compared to males $(p=0.0002)$.

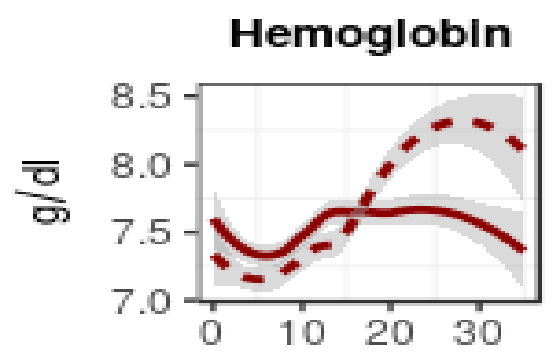

RBC

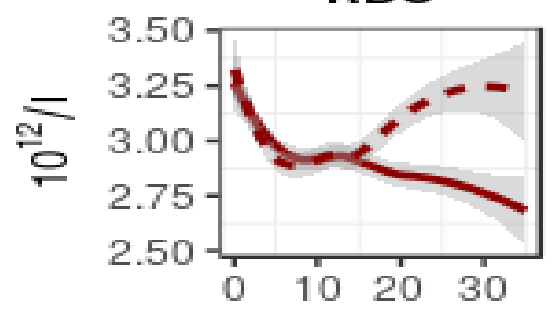

Platelet

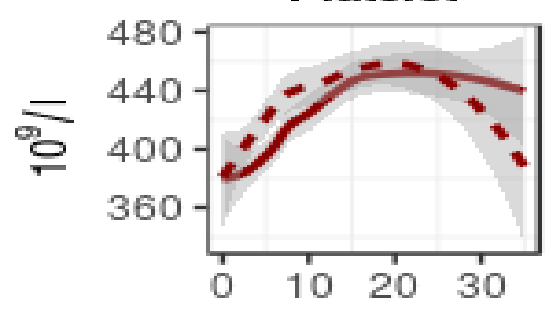

NeutraphIls

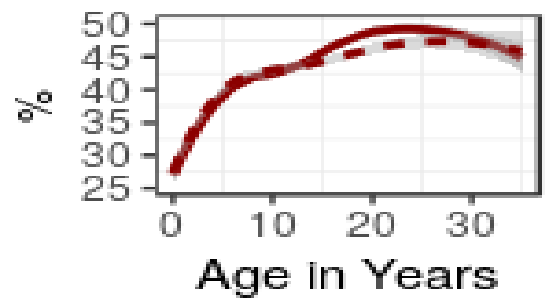

hbF

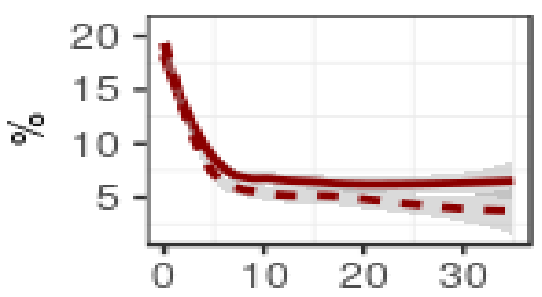

MCV

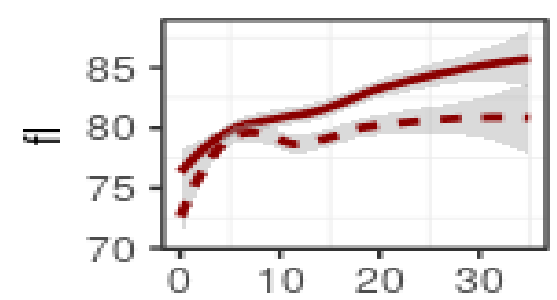

MPV

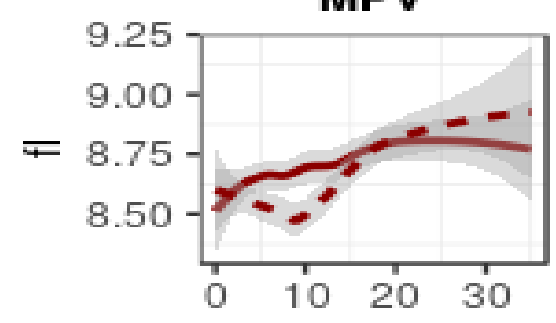

Eosinophlls

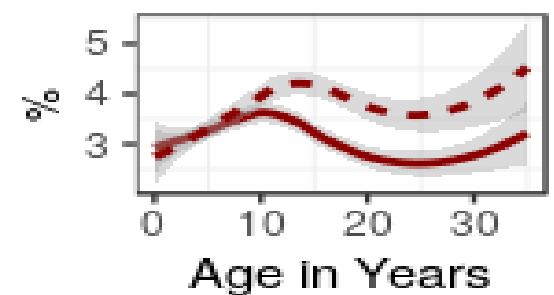

MCH

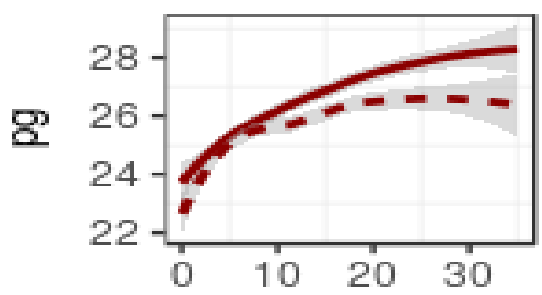

RDW

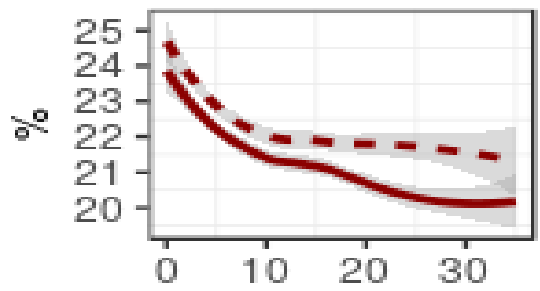

WBC

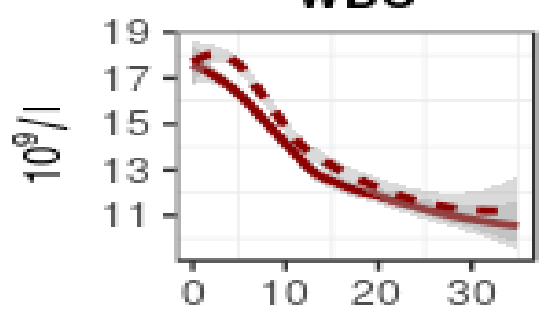

Monocytes

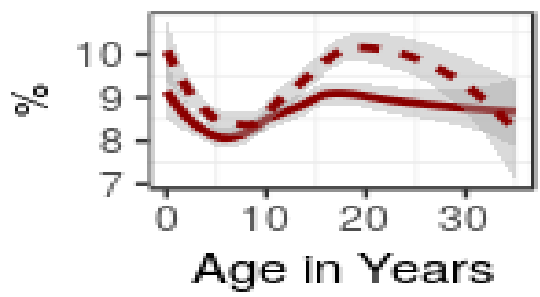

Figure 1. Smoothed mean curves for selected hematological parameters by sex. Solid lines denote the mean values for females and dashed lines denote the mean values for males.

Furthermore, the mean WBC was higher in males. However, the observed difference was not significant in infants ( $<1$ year) and adults ( $\geq 18$ years). WBC components, i.e., neutrophils and eosinophils, were evidently different in adolescents and adults when the sexual category analyzed their mean levels. The mean lymphocytes were higher in females though significantly different observed in the age groups of $<1$ and 5-11 years. More elevated mean monocytes were recorded in males, but the difference was not statistically significant in infants and children aged 5-11years. The mean basophils were comparable between males and females. 

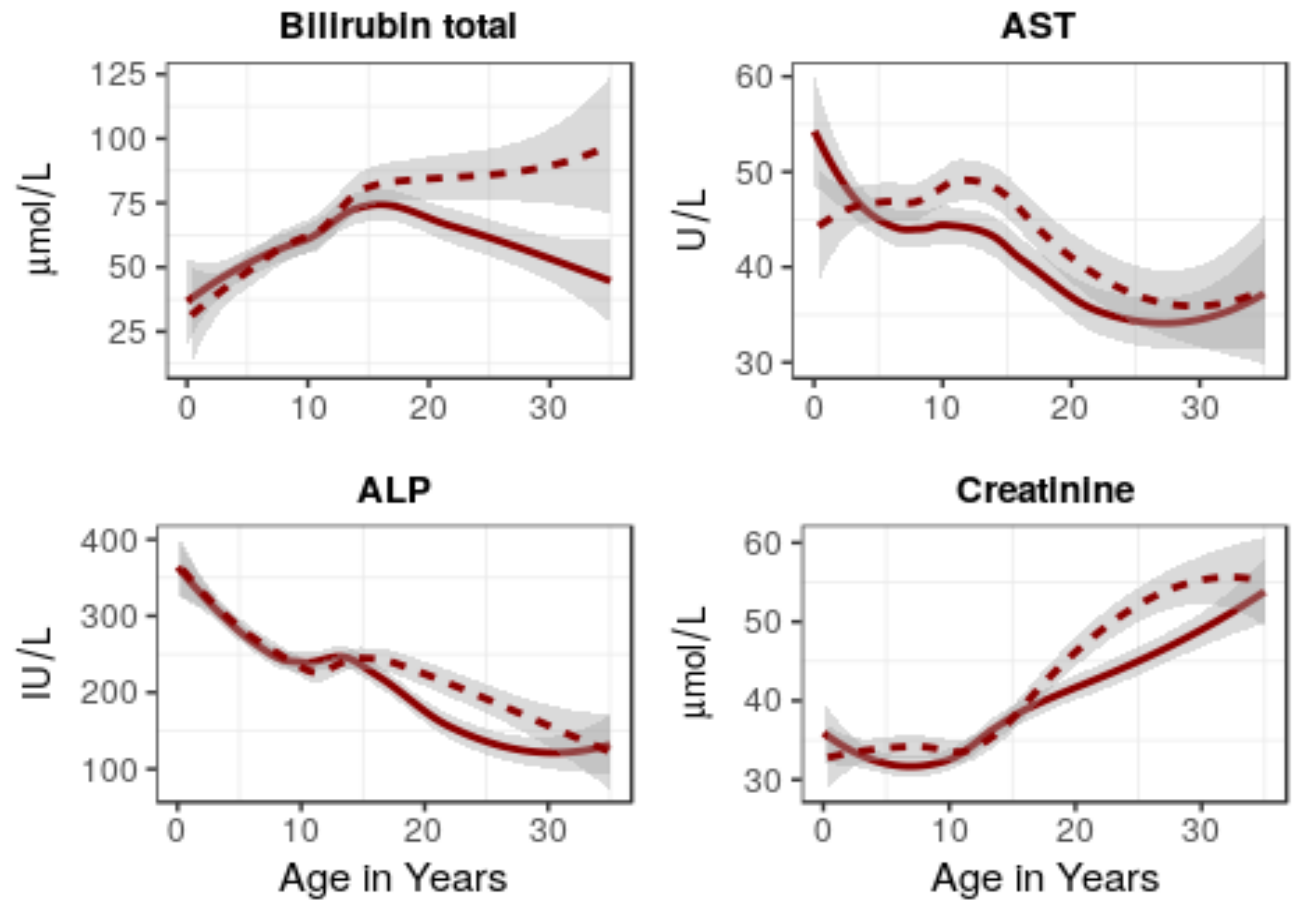

Figure 2.

mean curves for selected biochemical parameters by sex. Solid lines

denote the mean values for females and dashed lines denote the mean values for males.

\subsection{Biochemical parameters}

The variations of the mean values of biochemical parameters between males and females cases are shown in Table 3. While the mean bilirubin direct was comparable between males and females across age groups, the bilirubin total was significantly different in adults only ( $p=0.0243$ ). The differences mean AST of 12.2U/L, 3.3U/L, and 5.2U/L were different in infants, children aged 5-11 years, and adolescence, respectively. The mean ALP and creatinine were higher in males. Nevertheless, the differences were statistically significant in adults. Females were aged 12-17 years had significantly lower levels of LDH (by 75.5U/L).

Table 3 Steady-state biochemical reference values calculated as mean (mean $\pm 1.96 \mathrm{SD}$ ) in SCD categorized by age groups and sex.

\begin{tabular}{|c|c|c|c|c|c|c|c|}
\hline \multirow{2}{*}{$\begin{array}{l}\text { Age group } \\
\text { (years) }\end{array}$} & \multicolumn{2}{|r|}{ Females } & \multicolumn{2}{|r|}{ Males } & \multicolumn{2}{|r|}{ Combined } & \multirow[t]{2}{*}{ P-values* } \\
\hline & $\mathbf{n}$ & $\begin{array}{c}\text { Mean (Mean } \\
\pm 1.96 \mathrm{SD})\end{array}$ & $\mathbf{N}$ & $\begin{array}{c}\text { Mean (Mean } \\
\quad \pm 1.96 S D)\end{array}$ & $\mathbf{n}$ & $\begin{array}{c}\text { Mean (Mean } \\
\pm 1.96 S D)\end{array}$ & \\
\hline \multicolumn{8}{|c|}{ Bilirubin direct ( $\mu \mathrm{mol} / \mathrm{L})$} \\
\hline$<1$ & 13 & $9.9(0.8-119.4)$ & 17 & 8.6(1.2-59.6) & 30 & $9.2(1.0-80.2)$ & 0.6752 \\
\hline $1-4$ & 154 & $7.4(1.0-54.8)$ & 169 & $6.7(0.7-64.1)$ & 323 & $7.0(0.8-59.6)$ & 0.5992 \\
\hline $5-11$ & 308 & $7.8(1.0-56.6)$ & 353 & $7.6(1.1-53.3)$ & 661 & $7.7(1.1-54.8)$ & 0.5685 \\
\hline $12-17$ & 198 & $6.0(0.5-62.0)$ & 184 & $6.0(0.6-55.5)$ & 382 & $6.0(0.6-58.7)$ & 0.8477 \\
\hline$\geq 18$ & 166 & $6.7(0.9-48.4)$ & 106 & $6.1(0.6-61.2)$ & 272 & $6.5(0.7-53.3)$ & 0.7343 \\
\hline \multicolumn{8}{|c|}{ Bilirubin total $(\mu \mathrm{mol} / \mathrm{L})$} \\
\hline
\end{tabular}




\begin{tabular}{|c|c|c|c|c|c|c|c|}
\hline \multirow{2}{*}{$\begin{array}{l}\text { Age group } \\
\text { (years) }\end{array}$} & \multicolumn{2}{|r|}{ Females } & \multicolumn{2}{|r|}{ Males } & \multicolumn{2}{|r|}{ Combined } & \multirow[t]{2}{*}{ P-values* } \\
\hline & $\mathbf{n}$ & $\begin{array}{c}\text { Mean (Mean } \\
\pm 1.96 \mathrm{SD})\end{array}$ & $\mathbf{N}$ & $\begin{array}{c}\text { Mean (Mean } \\
\pm 1.96 S D)\end{array}$ & $\mathbf{n}$ & $\begin{array}{c}\text { Mean (Mean } \\
\pm 1.96 \mathrm{SD})\end{array}$ & \\
\hline$<1$ & 17 & $35.4(10.7-117.3)$ & 18 & $30.2(8.2-110.8)$ & 35 & 32.6(9.4-113.1) & 0.7165 \\
\hline $1-4$ & 153 & 39.5(11.4-136.8) & 158 & 36.4(11.4-116.4) & 311 & $37.9(11.4-126.2)$ & 0.2966 \\
\hline $5-11$ & 280 & $50.1(14.6-170.9)$ & 318 & $50.5(15.4-165.9)$ & 598 & $50.3(15.0-168.1)$ & 0.8201 \\
\hline $12-17$ & 162 & $59.4(15.6-225.7)$ & 160 & $58.0(11.5-291.3)$ & 322 & $58.7(13.4-257.4)$ & 0.8052 \\
\hline$\geq 18$ & 152 & $48.9(15.1-158.5)$ & 85 & 59.9(13.7-161.7) & 237 & $52.6(14.3-193.4)$ & 0.0243 \\
\hline \multicolumn{8}{|l|}{ AST (U/L) } \\
\hline$<1$ & 18 & 51.6(13.3-114.9) & 16 & 39.4(18.3-88.5) & 34 & $45.7(14.0-95.4)$ & 0.0180 \\
\hline $1-4$ & 156 & $44.8(20.1-79.4)$ & 162 & 46.2(21.2-80.7) & 318 & $45.5(20.7-80.0)$ & 0.2745 \\
\hline $5-11$ & 283 & $43.1(20.9-73.4)$ & 321 & $46.4(22.8-78.3)$ & 604 & $44.9(21.8-76.2)$ & 0.0029 \\
\hline $12-17$ & 165 & $40.7(16.2-76.4)$ & 161 & $45.9(19.8-82.8)$ & 326 & $43.2(17.7-80.0)$ & 0.0009 \\
\hline$\geq 18$ & 155 & 33.1(12.2-64.0) & 87 & $34.7(12.7-67.5)$ & 242 & 33.6(12.4-65.3) & 0.5586 \\
\hline \multicolumn{8}{|l|}{ ALP (IU/L) } \\
\hline$<1$ & 18 & $305.4(89.4-650.0)$ & 16 & $335.5(90.8-734.5)$ & 34 & $319.4(91.6-685.0)$ & 0.3427 \\
\hline $1-4$ & 158 & $290.2(119.0-563.7)$ & 163 & 300.1(106.5-577.8) & 321 & 297.6(112.7-570.6) & 0.8089 \\
\hline 5-11 & 285 & $235.7(86.1-458.9)$ & 322 & $240.2(107.0-426.6)$ & 607 & $238.1(96.6-442.3)$ & 0.4232 \\
\hline $12-17$ & 167 & 222.6(61.9-482.6) & 162 & $240.3(89.3-464.4)$ & 329 & 231.2(74.2-475.1) & 0.1035 \\
\hline$\geq 18$ & 154 & $125.1(30.0-285.3)$ & 89 & $158.3(35.8-367.9)$ & 243 & $136.8(30.5-319.5)$ & 0.0005 \\
\hline \multicolumn{8}{|l|}{ LDH (U/L) } \\
\hline$<1$ & 18 & 794.1(123.4-2047.8) & 16 & $829.4(234.0-1789.4)$ & 34 & 810.6(174.1-1913.9) & 0.9313 \\
\hline $1-4$ & 151 & $903.0(314.8-1794.2)$ & 159 & $946.9(352.7-1828.6)$ & 310 & $925.4(333.9-1812.0)$ & 0.2646 \\
\hline $5-11$ & 279 & $840.1(316.5-1614.4)$ & 316 & 819.2(236.5-1752.7) & 595 & $829.0(271.3-1690.2)$ & 0.5434 \\
\hline $12-17$ & 166 & $784.7(208.7-1728.8)$ & 159 & 860.2(282.7-1750.8) & 325 & $821.2(241.8-1744.1)$ & 0.0207 \\
\hline$\geq 18$ & 153 & 637.9(156.4-1444.6) & 87 & 714.9(131.8-1763.4) & 240 & 665.3(145.0-1563.8) & 0.2412 \\
\hline \multicolumn{8}{|c|}{ Creatinine $(\mu \mathrm{mol} / \mathrm{L})$} \\
\hline$<1$ & 18 & $31.7(6.7-75.3)$ & 18 & $32.8(17.4-52.9)$ & 36 & $32.2(10.8-65.0)$ & 0.9620 \\
\hline $1-4$ & 158 & $32.6(18.9-50.0)$ & 162 & 33.3(18.1-53.0) & 320 & $32.9(18.5-51.5)$ & 0.4853 \\
\hline 5-11 & 284 & $31.7(16.8-51.4)$ & 320 & $33.4(17.1-55.2)$ & 604 & 32.6(16.9-53.5) & 0.0547 \\
\hline $12-17$ & 167 & $35.1(18.4-62.3)$ & 168 & 37.8(16.1-63.1) & 335 & $36.4(17.2-62.7)$ & 0.2763 \\
\hline$\geq 18$ & 151 & $48.8(20.4-75.9)$ & 87 & $51.3(23.8-89.0)$ & 238 & $46.4(21.1-81.6)$ & 0.0002 \\
\hline
\end{tabular}

*Welch Two Sample t-test. SD; Standard deviation

\subsection{Laboratory parameters in SCD against the general population}

Table A1 compares values in SCD and that of the general Tanzanian population published elsewhere [11, 21, 22]. Individuals with SCD had significantly lower reference values for hemoglobin, $\mathrm{MCH}$, and lymphocytes. In comparison, values for platelets (except infants' group), WBC, bilirubin direct, bilirubin total, and AST were significantly higher when compared to that of the general 
population (almost $\mathrm{p}<0.0001$ in each case). MCHC, Neutrophils, platelets, and ALP in infants had estimated ranges comparable to the general population. It was interesting to observe that SCD children below five years had significantly higher MCV and creatinine reference values than that of the general population, but this changed for the older age groups where reference values from the general population were higher compared to those with SCD.

\section{Discussion}

This is example 2 of an equation: This study is the first to report reference values for patients with SCD at steady-state by age groups and by sex. Most laboratory parameters (platelets, white blood cells, bilirubin direct, bilirubin total, aspartate Aminotransferase, and alkaline phosphatase) were found to have higher values when compared to that of the general population, which was also different across the age groups. The reference ranges in the SCD were more or less similar between males and females. This indicates the importance of relevant reference values established using the Tanzania SCD population, as relying on reference intervals from other countries or that of the general population may lead to under or overestimating the disease condition.

The findings of 7.2-7.3g/dL hemoglobin concentrations in SCD children of under-five years were higher than $6.3-6.9 \mathrm{~g} / \mathrm{dL}$ reported by other researchers $[8$, 14,23 ] ; but lower than $8.1-9.3 \mathrm{~g} / \mathrm{dL}$ which have been reported in the cooperative study of sickle cell disease conducted in Western countries [24]. These observations of differences in hemoglobin may be due to the background of the development of malaria parasites in our environment [25].

Moreover, the lower steady-state hemoglobin concentration in SCD patients when compared to a healthy population can be explained by continual haemolysis of the red cells with a short survival rate of the erythrocytes in SCD individuals [15]. The mean of hemoglobin, $\mathrm{HbF}, \mathrm{MCH}, \mathrm{RBC}, \mathrm{MCV}$, RDW, eosinophils, bilirubin total, ALP, and creatinine were different between females and males, with most deviations happening after the age of 15 years. This is likely due to the start of the menstruation period which has also been associated with changes in levels of these parameters in female macaques [26].

The study reports significantly higher MCV values in children with less than five years when compared to that of the general population. These higher values in children agree with findings from another study [16]. It is reported that a higher mean corpuscular volume in children is due to folate deficiency [27]. Lacking vitamin B9 (resulting from not eating enough foods like leafy green vegetables, beans, and grains) may be worse, especially in underfives who are more dependent and may have lower compliance with supplementary folate.

The study found that the platelet counts values reported here are higher than those presented by [7]. There was a significant difference in SCD platelet levels in all age groups except in infants' group when the values were compared to that of the general population. After four years, platelet counts were gradually elevated in sickle cell groups but declined in the general population. The advancement of platelet count in sickle cell 
patients was the consequences of the deficiency splenic pooling of young active platelets [28].

The mean WBC values are more common among SCD patients compared to the general population. A previous study suggested that medications reactions and viral infections are factors that could increase the number of white cells in the blood in the SCD population [29]. Additionally, the evidence of elevated steady-state WBC had shown in SCD children during bacterial infection and vaso-occlusive crises [30]. Based on these findings, we believe that periodic evaluation of SCD patients' thorough physical examination will help identify those with high WBC so that appropriate measures will be taken to moderate causes related to high $\mathrm{WBC}$.

We reported normal alkaline phosphatase levels in infant patients ( $p=0.1998$ ), while other patients had shown a significant increase compared to the general population. As documented exhaustively elsewhere, upraised levels of alkaline phosphatase in SCD patients could result from persistent vaso-occlusive crises relating to the bones. However, these elevated values are expected in children who are still growing [31]. This was evident in this study.

Creatinine levels were considerably lower in the SCD patients after four years of age. Low creatinine values in SCD patients agreed with those reported in other studies $[32,33]$. It was reported that the decrease of creatinine in these patients was caused by smaller physical stature [34]. This finding is consistent with a study investigating uric acid, urea, and creatinine levels in SCD patients, which reported that the decline of creatinine in these patients resulted from muscle weakness [33].

This study finds the hematological and biochemical reference values for SCD for different age groups (infants, children, adolescents, and adults) by sex for SCD at a steady-state. We compared these parameters with population Tanzanian published data by keeping in mind that one would like to know the reference ranges for the general population. We believe that the reference ranges reported in this study will serve as a useful resource in monitoring the status and management of SCD patients, evaluation of interventions involving SCD patients, and the source of reference for scholars working in indistinguishable environments.

\subsection{Limitations}

Although the sample size was large in our study, the number of males and female patients in the infants' group was less than 120, as recommended elsewhere $[35,36]$. This may be the reason for the failure of a considerable number of parameters in this group to attain the significance level. Furthermore, the interpretation of laboratory results may have been limited when the variation of a specific group is minimal from that of the population. Therefore, the criterion of partitioning the population reference ranges into subgroups (males and females in our case) using a 
significant differences criterion may seem insufficient than utilizing the clinically physiological approach $[35,36]$.

Author Contributions: B.P.M., R.Z.S., and Jmakani designed the research study; methodology, B.P.M., R.Z.S., and A.D.F.; data collection and curation, R.Z.S., Jmgaya, D.S., F.T., U.M., E.B., S.N., Jmakani and B.P.M.; formal analysis, A.D.F. and B.P.M.; discussion of results; A.D.F., R.Z.S., B.P.M., and Jmakani; writing-original draft, A.D.F.; writing-review and editing A.D.F., R.Z.S., Jmgaya, D.S., F.T., U.M., E.B., S.N., Jmakani and B.P.M; Project administration and supervision, B.P.M., R.Z.S., and Jmakani. All authors have read and agreed to the final draft.

Funding: No funding was provided for this study. Original data collection was funded by the Wellcome Trust, UK (Fellowship Julie Makani 072064, 093727; Project grant 080025; Strategic award 084538; Principal Wellcome Trust Fellowship R W Snow 103602 and Government of United Republic of Tanzania.

Institutional Review Board Statement: The study was conducted according to the guidelines of the Muhimbili National Hospital (MNH) and approved by Muhimbili University of Health and Allied Science (MUHAS) ethical committee (MU/RP/AEC/VOL XI/33).

Informed Consent Statement: During data collection informed consent was obtained from all SCD patients or parents/guardians in the case of children and adolescents (below 18 years).

Data Availability Statement: The Muhimbili data sharing policy is not finalized. The data used to support the findings of this study are available from the institute upon reasonable request.

Competing interests: The authors declare that they have no competing interests.

\section{Appendix A:}

Table A1. Medians (95\% reference ranges) in selected laboratory parameters for SCD at steady-states compared to population published data in Tanzania.

\begin{tabular}{|l|l|l|l|l|}
\hline $\begin{array}{l}\text { Analytes } \\
\text { (Unit) }\end{array}$ & $\begin{array}{l}\text { Age group in years } \\
\text { (sex) }\end{array}$ & $\begin{array}{l}\text { SCD Patients } \\
\text { Median (2.5-97.5\%) }\end{array}$ & $\begin{array}{l}\text { General Population } \\
\text { Median (2.5-97.5\%) }\end{array}$ & P-values* \\
\hline \multirow{3}{*}{$\begin{array}{l}\text { Hemoglobin } \\
\text { g/dL) }\end{array}$} & $<1$ & $7.3(4.6-9.5)$ & $10.7(8.1-13.2)^{\dagger}$ & $<0.0001$ \\
\cline { 2 - 6 } & $1-4$ & $7.3(4.5-9.7)$ & $11.3(8.1-13.9)^{\dagger}$ & $<0.0001$ \\
\cline { 2 - 6 } & $5-11$ & $7.3(4.9-9.4)$ & $12.6(10.3-14.7)^{\dagger}$ & $<0.0001$ \\
\hline & $12-17(\mathrm{~F})$ & $7.6(5.3-9.7)$ & $13.0(10.0-14.9)^{\dagger}$ & $<0.0001$ \\
\hline & $12-17(\mathrm{M})$ & $7.4(5.0-9.8)$ & $13.7(10.8-17.0)^{\dagger}$ & $<0.0001$ \\
\hline & $\geq 18(\mathrm{~F})$ & $7.6(5.1-10.0)$ & $13.5(11.1-15.7)^{\ddagger}$ & $<0.0001$ \\
\hline & $\geq 18(\mathrm{M})$ & $8.3(4.7-11.0)$ & $15.4(13.7-17.7)^{\ddagger}$ & $<0.0001$ \\
\cline { 2 - 6 } & $\geq 18(\mathrm{M} \& \mathrm{~F})$ & $7.9(4.8-10.6)$ & $14.7(11.7-17.2)^{\ddagger}$ & $<0.0001$ \\
\hline
\end{tabular}




\begin{tabular}{|c|c|c|c|c|}
\hline $\begin{array}{l}\text { Analytes } \\
\text { (Unit) }\end{array}$ & $\begin{array}{l}\text { Age group in years } \\
\text { (sex) }\end{array}$ & $\begin{array}{l}\text { SCD Patients } \\
\text { Median (2.5-97.5\%) }\end{array}$ & $\begin{array}{l}\text { General Population } \\
\text { Median }(2.5-97.5 \%)\end{array}$ & P-values* \\
\hline \multirow[t]{4}{*}{ MCH (pg) } & - & - & - & - \\
\hline & $\geq 18(\mathrm{~F})$ & $28.0(19.9-34.2)$ & $30.0(23.1-33.2) \ddagger$ & $<0.0001$ \\
\hline & $\geq 18(\mathrm{M})$ & 26.6(19.9-33.1) & $29.3(24.2-33.1) \ddagger$ & $<0.0001$ \\
\hline & $\geq 18(\mathrm{M} \& \mathrm{~F})$ & $27.5(19.9-34.0)$ & $29.7(23.6-33.1)^{\ddagger}$ & $<0.0001$ \\
\hline \multirow[t]{4}{*}{ MCHC (g/dL) } & - & - & - & - \\
\hline & $\geq 18(\mathrm{~F})$ & $32.8(29.9-36.1)$ & $33.3(30.6-35.1)^{\ddagger}$ & $<0.0001$ \\
\hline & $\geq 18(\mathrm{M})$ & $32.6(30.0-36.5)$ & $32.7(30.4-34.8) \ddagger$ & 0.9239 \\
\hline & $\geq 18(\mathrm{M} \& \mathrm{~F})$ & $32.8(30.0-36.3)$ & $33.0(30.6-34.9) \ddagger$ & 0.9856 \\
\hline \multirow[t]{8}{*}{$\operatorname{MCV}(f L)$} & $<1$ & $74.0(53.4-91.7)$ & $71.0(53.3-96.6)^{\dagger}$ & $<0.0001$ \\
\hline & $1-4$ & $79.0(59.7-95.3)$ & $75.0(54.7-91.6)^{+}$ & $<0.0001$ \\
\hline & $5-11$ & $80.0(60.5-98.0)$ & $82.5(66.0-90.0)^{+}$ & $<0.0001$ \\
\hline & $12-17(\mathrm{~F})$ & $82.0(64.3-99.1)$ & $84.9(62.2-94.5)^{\dagger}$ & $<0.0001$ \\
\hline & $12-17(\mathrm{M})$ & $79.0(63.4-93.5)$ & $82.0(63.2-91.0)^{\dagger}$ & $<0.0001$ \\
\hline & $\geq 18(\mathrm{~F})$ & $84.4((64.0-102.8)$ & $89.5(77.7-97.9) \ddagger$ & $<0.0001$ \\
\hline & $\geq 18(\mathrm{M})$ & $81.7(62.8-99.2)$ & $89.3(76.4-98.8) \ddagger$ & $<0.0001$ \\
\hline & $\geq 18(\mathrm{M} \& \mathrm{~F})$ & 83.3(63.0-101.4) & $89.4(77.6-98.1) \ddagger$ & $<0.0001$ \\
\hline \multirow{8}{*}{\begin{tabular}{|l|} 
Platelet Count \\
$\left(\times 10^{9} / 1\right)$
\end{tabular}} & $<1$ & $399(135-724)$ & $384(25-708)^{+}$ & 0.2923 \\
\hline & $1-4$ & $376(104-758)$ & $359(79-650)^{+}$ & 0.0087 \\
\hline & $5-11$ & $432(126-762)$ & $320(94-530)^{+}$ & $<0.0001$ \\
\hline & $12-17(\mathrm{~F})$ & $450(132-771)$ & $282(107-482)^{+}$ & $<0.0001$ \\
\hline & $12-17(\mathrm{M})$ & $445(179-808)$ & $271(119-458)^{\dagger}$ & $<0.0001$ \\
\hline & $\geq 18(\mathrm{~F})$ & $448(143-738)$ & $271(151-425) \ddagger$ & $<0.0001$ \\
\hline & $\geq 18(\mathrm{M})$ & $435(179-777)$ & $224(147-356) \ddagger$ & $<0.0001$ \\
\hline & $\geq 18(\mathrm{M} \& \mathrm{~F})$ & $439(148-765)$ & $244(150-359) \ddagger$ & $<0.0001$ \\
\hline \multirow{5}{*}{$\begin{array}{r}\text { WBC count } \\
\left(\times 10^{9} / 1\right)\end{array}$} & $<1$ & 13.8(7.8-33.7) & $9.2(5.0-17.3)^{+}$ & $<0.0001$ \\
\hline & $1-4$ & $17.2(8.2-30.1)$ & $7.7(3.7-13.2)^{+}$ & $<0.0001$ \\
\hline & 5-11 & $14.7(7.7-25.3)$ & $5.6(3.7-9.1)^{+}$ & $<0.0001$ \\
\hline & $12-17$ & 12.6(6.8-21.1) & $5.3(3.2-10.3)^{+}$ & $<0.0001$ \\
\hline & $\geq 18$ & - & - & - \\
\hline \multirow{4}{*}{$\begin{array}{c}\text { Lymphocytes } \\
(\%)\end{array}$} & - & - & - & - \\
\hline & $\geq 18(\mathrm{~F})$ & $37.3(19.7-53.1)$ & $40.4(20.9-56.7)^{\ddagger}$ & $<0.0001$ \\
\hline & $\geq 18(\mathrm{M})$ & $36.8(16.9-54.2)$ & $40.8(20.8-57.3)^{\ddagger}$ & $<0.0001$ \\
\hline & $\geq 18(\mathrm{M} \& \mathrm{~F})$ & $37.1(18.2-54.6)$ & $40.4(20.8-56.7)^{\ddagger}$ & $<0.0001$ \\
\hline \multirow{4}{*}{$\begin{array}{l}\text { Neutrophils } \\
(\%)\end{array}$} & - & - & - & - \\
\hline & $\geq 18(\mathrm{~F})$ & $48.6(30.4-67.3)$ & $48.4(32.5-71.3) \ddagger$ & 0.7035 \\
\hline & $\geq 18(\mathrm{M})$ & $46.8(26.8-69.7)$ & $47.3(31.7-69.3) \ddagger$ & 0.4763 \\
\hline & $\geq 18(\mathrm{M} \& \mathrm{~F})$ & $47.6(28.5-69.6)$ & $48.1(32.0-69.1)^{\ddagger}$ & 0.6826 \\
\hline \multirow{4}{*}{$\begin{array}{l}\text { Bilirubin di- } \\
\text { rect }(\mu \mathrm{mol} / \mathrm{L})\end{array}$} & & - & - & - \\
\hline & $\geq 18(\mathrm{~F})$ & $8.1(0.0-23.4)$ & $1.81(0.70-5.83) \ddagger$ & $<0.0001$ \\
\hline & $\geq 18(\mathrm{M})$ & $7.5(0.0-32.9)$ & $2.53(0.93-8.43) \ddagger$ & $<0.0001$ \\
\hline & $\geq 18(\mathrm{M} \& \mathrm{~F})$ & $7.8(0.0-29.3)$ & $2.21(0.72-8.22)^{\ddagger}$ & $<0.0001$ \\
\hline
\end{tabular}




\begin{tabular}{|c|c|c|c|c|}
\hline $\begin{array}{l}\text { Analytes } \\
\text { (Unit) }\end{array}$ & $\begin{array}{l}\text { Age group in years } \\
\text { (sex) }\end{array}$ & $\begin{array}{l}\text { SCD Patients } \\
\text { Median }(2.5-97.5 \%)\end{array}$ & $\begin{array}{l}\text { General Population } \\
\text { Median (2.5-97.5\%) }\end{array}$ & P-values* \\
\hline \multicolumn{2}{|c|}{ Bilirubin total $<1$} & $32.2(10.4-90.5)$ & $4(2-19)^{P}$ & $<0.0001$ \\
\hline \multirow[t]{7}{*}{$(\mu \mathrm{mol} / \mathrm{L})$} & $1-4$ & $36.9(7.8-132.3)$ & $4(2-9)^{\mathbb{P}}$ & $<0.0001$ \\
\hline & $5-11$ & $46.4(1.9-184.0)$ & $4(2-11)^{\mathbb{P}}$ & $<0.0001$ \\
\hline & $12-17(\mathrm{~F})$ & $55.8(16.7-237.9)$ & $6(3-22)^{P}$ & $<0.0001$ \\
\hline & $12-17(\mathrm{M})$ & $57.8(10.5-295.2)$ & $6(2-15)^{P}$ & $<0.0001$ \\
\hline & $\geq 18(\mathrm{~F})$ & $45.5(10.2-170.9)$ & $13.9(6.0-42.0) \ddagger$ & $<0.0001$ \\
\hline & $\geq 18(\mathrm{M})$ & $52.6(12.9-251.8)$ & $10.0(4.5-31.3) \ddagger$ & $<0.0001$ \\
\hline & $\geq 18(\mathrm{M} \& \mathrm{~F})$ & $48.6(10.9-216.0)$ & $11.5(5.2-41.0) \ddagger$ & $<0.0001$ \\
\hline \multirow[t]{8}{*}{$\operatorname{AST}(\mathrm{U} / \mathrm{L})$} & $<1$ & $46.0(11.8-101.2)$ & $38(26-65)^{\mathbb{P}}$ & 0.0057 \\
\hline & $1-4$ & $43.0(24.4-89.3)$ & $36(27-55)^{P}$ & $<0.0001$ \\
\hline & $5-11$ & $43.5(24.0-86.8)$ & $31(21-51)^{P}$ & $<0.0001$ \\
\hline & $12-17(\mathrm{~F})$ & $38.0(20.0-86.2)$ & $23(17-36)^{P}$ & $<0.0001$ \\
\hline & $12-17(\mathrm{M})$ & $44.0(22.5-90.0)$ & $27(19-42)^{\mathbb{P}}$ & $<0.0001$ \\
\hline & $\geq 18(\mathrm{~F})$ & $32.0(16.7-66.0)$ & $20.1(13.5-35.2) \ddagger$ & $<0.0001$ \\
\hline & $\geq 18(\mathrm{M})$ & $31.0(18.0-78.9)$ & $28.2(15.2-53.4) \ddagger$ & $<0.0001$ \\
\hline & $\geq 18(\mathrm{M} \& \mathrm{~F})$ & $31.0(18.0-61.8)$ & $22.8(14.3-48.1)^{\ddagger}$ & $<0.0001$ \\
\hline \multirow[t]{8}{*}{ ALP (IU/L) } & $<1$ & $322(53-586)$ & $307(164-589)^{\mathbb{P}}$ & 0.1998 \\
\hline & $1-4$ & $288(120-600)$ & $253(153-410)^{\mathbb{P}}$ & $<0.0001$ \\
\hline & $5-11$ & $237(110-468)$ & $278(174-460)^{P}$ & $<0.0001$ \\
\hline & $12-17(\mathrm{~F})$ & $216(71-556)$ & $191(68-498)^{\mathbb{P}}$ & $<0.0001$ \\
\hline & $12-17(\mathrm{M})$ & $229(122-488)$ & $302(124-537)^{\mathbb{P}}$ & $<0.0001$ \\
\hline & $\geq 18(\mathrm{~F})$ & $115(46-310)$ & $85.0(45.4-170.4) \ddagger$ & $<0.0001$ \\
\hline & $\geq 18(\mathrm{M})$ & $153(59-408)$ & $75.4(45.3-155.0)^{\ddagger}$ & $<0.0001$ \\
\hline & $\geq 18(\mathrm{M} \& \mathrm{~F})$ & $123(47-396)$ & $80.0(45.6-158.4) \ddagger$ & $<0.0001$ \\
\hline \multirow{8}{*}{$\begin{array}{l}\text { Creatinine } \\
(\mu \mathrm{mol} / \mathrm{L})\end{array}$} & $<1$ & $32.5(13.2-57.1)$ & $18(12-28)^{P}$ & $<0.0001$ \\
\hline & $1-4$ & $32.0(20.0-56.0)$ & $24(15-50)^{P}$ & $<0.0001$ \\
\hline & 5-11 & $32.0(18.0-56.0)$ & $36(24-49)^{P}$ & $<0.0001$ \\
\hline & $12-17(\mathrm{~F})$ & $35.0(19.1-68.0)$ & $45(32-64)^{P}$ & $<0.0001$ \\
\hline & $12-17(\mathrm{M})$ & $34.0(18.0-69.1)$ & $48(36-80)^{P}$ & $<0.0001$ \\
\hline & $\geq 18(\mathrm{~F})$ & $42.5(25.0-86.0)$ & $56(40-81) \ddagger$ & $<0.0001$ \\
\hline & $\geq 8(\mathrm{M})$ & $51.0(26.3-83.4)$ & $69(48-96) \ddagger$ & $<0.0001$ \\
\hline & $\geq 18(\mathrm{M} \& \mathrm{~F})$ & $44.7(25.0-85.3)$ & $62(42-90) \ddagger$ & $<0.0001$ \\
\hline
\end{tabular}

* Mann Whitney U test for significance difference. $\quad$ +Obtained from [11]; $\mathbb{P}$ from [21] and and [21] age ranges were listed as; $<12$ months, 1 to $<5$ years, 5 to $<13$ years, 13 to $<18$ years (M), 13 to $<18$ years (F). In [22] age range is 19 to 48 years. 


\section{References}

1. Modell B, Darlison M (2008) Global epidemiology of haemoglobin disorders and derived service indicators. Bulletin of the World Health Organization 86:480-487

2. Makani J, Komba AN, Cox SE, et al (2010) Malaria in patients with sickle cell anemia: burden, risk factors, and outcome at the outpatient clinic and during hospitalization. Blood 115:215-220

3. Makani J, Cox SE, Soka D, et al (2011) Mortality in sickle cell anemia in Africa: a prospective cohort study in Tanzania. PloS one 6:e14699

4. Tluway F, Makani J (2017) Sickle cell disease in Africa : an overview of the integrated approach to health, research, education and advocacy in Tanzania, 2004-2016. Br J Haematol 177:919-929

5. Bhatia M, Walters MC (2008) Hematopoietic cell transplantation for thalassemia and sickle cell disease: Past, present and future. Bone Marrow Transplantation 41:109-117

6. Vermylen C, Cornu G, Ferster A, et al (1998) Haematopoietic stem cell transplantation for sickle cell anaemia: The first 50 patients transplanted in Belgium. Bone Marrow Transplantation 22:1-6

7. Akinbami A, Dosunmu A, Adediran A, Oshinaike O, Adebola P, Arogundade O (2012) Haematological values in homozygous sickle cell disease in steady state and haemoglobin phenotypes AA controls in Lagos, Nigeria. BMC research notes 5:396

8. Iheanacho O (2015) Haematological Parameters of Adult and Paediatric Subjects with Sickle Cell Disease in Steady State, in Benin City, Nigeria. International Blood Research \& Reviews 3:171-177

9. Ballas SK, Smith ED (1992) Red blood cell changes during the evolution of the sickle cell painful crisis. Blood 79:2154-2163

10. Powars DR, Schroeder WA, Weiss JN, Chan LS, Azen SP (1980) Lack of influence of fetal hemoglobin levels or erythrocyte indices on the severity of sickle cell anemia. Journal of Clinical Investigation 65:732-740

11. Buchanan AM, Muro FJ, Gratz J, et al (2010) Establishment of haematological and immunological reference values for healthy Tanzanian children in Kilimanjaro Region. Tropical Medicine \& International Health 15:10111021

12. Lugada ES, Mermin J, Kaharuza F, Ulvestad E, Were W, Langeland N, Asjo B, Malamba S, Downing R (2004) Population-based hematologic and immunologic reference values for a healthy Ugandan population. Clinical and Diagnostic Laboratory Immunology 11:29-34

13. Quintó L, Aponte JJ, Sacarlal J, et al (2006) Haematological and biochemical indices in young African children: in search of reference intervals. Tropical Medicine \& International Health 11:1741-1748

14. Akodu SO, Njokanma OF, AdeoluKehinde O (2015) Erythrocyte indices in Pre-School Nigerian children with sickle cell anaemia in steady state. International Journal of Hematology-Oncology and Stem Cell Research 9:5-9

15. Emmanuelchide O, Charle O, Uchenna O (2011) Hematological parameters in association with outcomes in sickle cell anemia patients0. Indian Journal of Medical Sciences 65:393

16. Omoti CE (2005) Haematological values in sickle cell anaemia in steady state and during vaso-occlusive crisis in Benin City, Nigeria. Ann Afr Med 4:62-67

17. Katayev A, Balciza C, Seccombe DW (2010) Establishing reference intervals for clinical laboratory test results: is there a better way? American journal of clinical pathology 133:180-186

18. Ballas SK (2012) More definitions in sickle cell disease: Steady state v base line data. American Journal of Hematology 87:338-338

19. Makani J, Tluway F, Makubi A, et al (2018) A ten year review of the sickle cell program in Muhimbili National Hospital, Tanzania. BMC Hematology 18:1-13 
20. Bertholf RL (2006) Statistical Methods for Establishing and Validating Reference Intervals. Laboratory Medicine 37:306-310

21. Buchanan AM, Fiorillo SP, Omondi MW, Cunningham CK, Crump JA (2015) Establishment of biochemistry reference values for healthy Tanzanian infants, children and adolescents in Kilimanjaro Region. Tropical Medicine \& International Health 20:1569-1577

22. Saathoff E, Schneider P, Kleinfeldt V, Geis S, Haule D, Maboko L, Samky E, Souza M de, Robb M, Hoelscher M (2008) Laboratory reference values for healthy adults from southern Tanzania. Tropical Medicine \& International Health 13:612-625

23. Abbas M (2014) Haematological parameters in Sudanese children with sickle cell disease. Am J Res Com 2:2032

24. Brown AK, Sleeper LA, Miller ST, Pegelow CH, Gill FM, Waclawiw MA (1994) Reference Values and Hematologic Changes From Birth to 5 Years in Patients With Sickle Cell Disease. Archives of pediatrics \& adolescent medicine 148:796-804

25. Desai MR, Terlouw DJ, Kwena AM, et al (2005) Factors associated with hemoglobin concentrations in pre-school children in western Kenya: Cross-sectional studies. American Journal of Tropical Medicine and Hygiene 72:4759

26. Perigard CJ, Parrula MCM, Larkin MH, Gleason CR (2016) Impact of menstruation on select hematology and clinical chemistry variables in cynomolgus macaques. Veterinary clinical pathology 45:232-243

27. Irwin J, Kirchner J (2008) Anemia in children. Nippon rinsho Japanese journal of clinical medicine 66:544-547

28. Allen B, Schwartz D (1972) The Splenic Platelet Reservoir in Sickle Cell Anemia. Blood 40:678-683

29. Riley LK, Rupert J (2015) Evaluation of Patients with Leukocytosis. American family physician 92:1004-1011

30. Buchanan GR, Glader BE (1978) Leukocyte Counts in Children With Sickle Cell Disease: Comparative Values in the Steady State, Vaso-occlusive Crisis, and Bacterial Infection. American Journal of Diseases of Children. https://doi.org/10.1001/archpedi.1978.02120290068013

31. Kotila T, Adedapo K, Adedapo A, Oluwasola O, Fakunle E, Brown B (2019) Liver dysfunction in steady state sickle cell disease. Annals of Hepatology 4:261-263

32. Al-Ali AK, Ahmed MAM, Qaw F. S, Al-Fadel Saleh M, Al-Bashir A (1995) Uric Acid, Creatinine and Urea in Normal , Glucose-6-Phosphate Dehydrogenase-Deficient and Hb S S Saudi Subjects. Acta haematologica 94:114116

33. Al-naama LM, Emad A-S, Al-Sadoon TA (2000) Levels of Uric Acid, Urea and Creatinine in Iraqi Children with Sickle Cell Disease. Journal-Pakistan Medical Association 50:98-101

34. Odonkor PO, Addae SK, Yamamoto S, Apatu RS (1984) Effect of dietary nitrogen on urinary excretion of nonprotein nitrogen in adolescent sickle cell patients. Human nutrition Clinical nutrition 38:23-29

35. Reed AH, Henry RJ, Mason WB (1971) Influence of statistical method used on the resulting estimate of normal range. Clinical Chemistry 17:275-284

36. Harris EK, Wong ET, Shaw ST (1991) Statistical criteria for separate reference intervals: Race and gender groups in creatine kinase. Clinical Chemistry 37:1580-1582 